\title{
Numerical study for tensile strength prediction of unidirectional carbon fiber-reinforced composite considering fiber surface stress concentration
}

\author{
Go YAMAMOTO* and Tomonaga OKABE*,** \\ *Department of Aerospace Engineering, Tohoku University, \\ 6-6-01 Aramaki-Aza-Aoba, Aoba-ku, Sendai 980-8579, Japan \\ E-mail: yamamoto@plum.mech.tohoku.ac.jp \\ **Department of Materials Science and Engineering, University of Washington, \\ Seattle, WA 98195, U.S.A.
}

Received: 8 January 2019; Revised: 21 March 2019; Accepted: 16 April 2019

\begin{abstract}
The accurate tensile strength prediction of unidirectional carbon fiber-reinforced plastic composites (UD composites) requires approximate determination of the stress concentration on surviving fibers around a fiber break point. Here the stress concentrated on the intact fiber surface was determined by implementing double-fiber fragmentation tests in combination with a spring element model (SEM) simulation. The double-fiber fragmentation composites and the UD composites were elaborated with a T1100G-type carbon fiber and epoxy material, and tested to validate the proposed prediction method. The size scaling results, implementing a bimodal Weibull distribution for the statistical distribution of fiber strength, coupled with the results of the SEM simulation, designed to take into account the surface stress concentration, were reasonably consistent with the experimental data on the tensile strengths of the UD composites. Then, the proposed strength prediction procedure was applied to investigate the effects of the bimodal Weibull scale and shape parameters on the tensile strength of the UD composites. It was revealed that the degree of stress concentrated on the surface of fibers can be changed by modifying the bimodal Weibull shape and scale parameters. However, the carbon fiber with an improved scale parameters of $20 \%$ displayed enhancement to the composite strength by factor of $\sim 1.07$, and with an improved shape parameters of $20 \%$ showed enhancement by factor of $\sim 1.04$, indicating that the degree of enhancement in the tensile strength of the UD composite was limited.
\end{abstract}

Keywords : Carbon fiber, Polymer-matrix composite (PMC), Fragmentation, Strength, Stress concentration

\section{Introduction}

Fiber-reinforced polymer-matrix composites (PMC) have been widely used across industries including aerospace, wind energy, pressure vessel, and automotive applications. Carbon fiber-reinforced plastic (CFRP), a typical class of composite materials, is increasingly being used as lightweight and high-stiffness materials in the above-mentioned applications. The processes of determining the potential amount of weight that can be saved requires that the fracture properties of the CFRPs in the fiber axis direction be a major consideration for designing composite structures. The mechanical modeling of failure of unidirectional carbon fiber-reinforced composites (UD composites) is a useful first step for understanding of real composites including multi-ply laminates and woven composites. Thus, improving the accuracy of tensile strength prediction methods continues to be central to CFRP composite research.

A number of extent researches aimed at finding ways to accurately predict the tensile strength of the UD composites (Scott et al., 2011; Swolfs et al., 2015; Watanabe et al., 2018). A local load sharing (LLS) model developed by Curtin for the numerical simulation of fracture behavior in fiber-reinforced composites has been widely applied to predict the ultimate tensile strength of UD composites (Curtin et al., 2000). The author defined the force redistribution concept of the LLS as follows; the perturbation of load caused by a fiber failure is not distributed uniformly among the 
remaining fibers, but rather is carried more largely by adjacent surviving fibers. Thus, when one fiber breaks the load that it was carrying is transferred principally to the surviving neighbors, increasing the stress concentration of these fibers relative to the others. Consequently, increased probability of failure of such fibers leads to the formation of clusters of broken fibres, leading to final failure of the composites. Although the stress concentration on an intact fiber surface around a fiber break point has been widely recognized as an important factor controlling the tensile strength of UD composites, the strength prediction of such composite taking into account the stress concentration due to the fiber failure is so far from satisfactory.

In this study, we considered the stress concentration on an intact fiber surface caused by a fracture site in a neighboring broken fiber into our prediction of the ultimate tensile strengths of the UD composites. The stress concentrated on the intact fiber surface was determined by implementing double-fiber fragmentation tests in combination with a spring element model (SEM) simulation. We chose herein to apply a bimodal Weibull distribution as the statistical distribution of fiber strength, based on the knowledge by Watanabe et al. (Watanabe et al., 2018). The acquired stress concentration factors were subsequently implemented to obtain the tensile strength prediction of the UD composites. The double-fiber fragmentation composites and the UD composites were elaborated with a T1100G-type carbon fiber and epoxy material, and tested to validate the proposed prediction method. The size scaling results obtained in conjunction with the results obtained from the SEM simulation were reasonably consistent with the experimental data on the tensile strengths of the UD composites. We have also systematically investigated the effects of the bimodal Weibull scale and shape parameters on the tensile strength of the UD composites.

\section{Analytical and experimental procedures \\ 2.1 Analytical model}

Monte-Carlo methods were implemented in the SEM simulation to determine the stress concentrated on the intact fiber surface around the fiber break points. The details of model preparation and simulation procedures can be found in the reference (Okabe et al., 2005; Watanabe et al., 2018). In brief, the SEM comprises longitudinal and transverse spring elements in a three-dimensional hexagonal arrangement. The longitudinal elements represent carbon fibers that exclusively carry the tensile load, while the transverse elements indicate the matrix that only sustains the shearing load. The stiffness matrices of the fiber element $\boldsymbol{K}_{\mathrm{L}}^{\mathrm{e}}$ and matrix element $\boldsymbol{K}_{\mathrm{T}}^{\mathrm{e}}$ are separately calculated as follows:

$$
\begin{aligned}
& \boldsymbol{K}_{\mathrm{L}}^{\mathrm{e}}=\pi R^{2} \int_{0}^{l} \boldsymbol{B}_{\mathrm{L}}^{\mathrm{eT}} E_{\mathrm{f}} \boldsymbol{B}_{\mathrm{L}}^{\mathrm{e}} \mathrm{d} z, \\
& \boldsymbol{K}_{\mathrm{T}}^{\mathrm{e}}=\frac{\pi R l}{3} \int_{0}^{l} \boldsymbol{B}_{\mathrm{T}}^{\mathrm{eT}} G \boldsymbol{B}_{\mathrm{T}}^{\mathrm{e}} \mathrm{d} r, \\
& \boldsymbol{B}_{\mathrm{L}}^{\mathrm{e}}=\left[\frac{1}{l}-\frac{1}{l}\right], \\
& \boldsymbol{B}_{\mathrm{T}}^{\mathrm{e}}=\left[\frac{1}{d}-\frac{1}{d}\right],
\end{aligned}
$$

where $\mathrm{L}$ and $\mathrm{T}$ indicate the longitudinal and transverse directions of the elements, respectively, $R$ is the radius of the carbon fiber $(=2.7 \mu \mathrm{m}), E_{\mathrm{f}}$ is the Young's modulus of the carbon fiber $(=324 \mathrm{GPa}), G_{\mathrm{m}}$ is the shear modulus of the epoxy material $(=1.4 \mathrm{GPa})$, and $l$ and $d$ are the spring element lengths along the longitudinal and transverse directions, respectively. The experimentally observed average interfiber spacings in the double-fiber fragmentation composites was approximately $0.01 \mathrm{~mm}$. Therefore, $d$ was set to 0.01 to analyze the results of the double-fiber fragmentation tests. Conversely, a hexagonal close-packed arrangement was assumed for the analysis of the full composite simulation and $d$ can be calculated as follows:

$$
d=R\left(\sqrt{2 \pi / \sqrt{3} V_{\mathrm{f}}}-2\right)
$$

where $V_{\mathrm{f}}$ is the fiber volume fraction in the full composite model $(=57 \%)$. In this study, we considered the situation where the fiber axial stress within the stress recovery region can be described as a linear function of the distance from 
the fiber breaking point, as is given by the following equation (Okabe et al., 2015):

$$
\sigma_{\mathrm{s}}=2 \tau_{\mathrm{s}} D_{\mathrm{s}} / \mathrm{R}
$$

where $\sigma_{\mathrm{s}}$ is the fiber axial stress within the stress recovery region, $\tau_{\mathrm{s}}$ is the interfacial shear stress, and $D_{\mathrm{s}}$ is the distance from the fiber breaking point. Therefore, the force balance to describe the overall system is given as follows:

$$
\left[\sum_{e=1}^{N \mathrm{f}-N \mathrm{~b}-N \mathrm{p}} \boldsymbol{K}_{\mathrm{L}}^{\mathrm{e}}-\sum_{e=1}^{N \mathrm{~m}} \boldsymbol{K}_{\mathrm{T}}^{\mathrm{e}}\right] \boldsymbol{u}+\sum_{e=1}^{N \mathrm{p}} \pi R^{2} \int_{0}^{l} \boldsymbol{B}_{\mathrm{L}}^{\mathrm{eT}} \sigma_{\mathrm{s}} \mathrm{d} z=\boldsymbol{f}
$$

where $N_{\mathrm{f}}$ and $N_{\mathrm{m}}$ are the number of fiber and matrix elements, respectively, $N_{\mathrm{b}}$ is the number of broken fiber, and $N_{\mathrm{p}}$ is the number of fiber elements in the plastic deformation region.

The statistical distribution of fiber strength was determined using both the single-fiber tensile testing and single-fiber fragmentation testing (Okabe et al., 2002; Watanabe et al, 2014). Table 1 shows the Weibull parameters implemented to construct a bimodal Weibull distribution of the T1100G carbon fiber. The fiber breakage probability $P_{\mathrm{f}}$ $(\sigma)$ under the conditions that the fiber is subjected to stress $\sigma$ at the surface area $\mathrm{S}_{\mathrm{f}}\left(=2 \pi R L_{\mathrm{f}}\right)$ is given as follows:

$$
P_{\mathrm{f}}(\sigma)=1-\exp \left\{-\frac{S_{\mathrm{f}}}{S_{\mathrm{f}, 0}}\left(\frac{\sigma}{\sigma_{01}}\right)^{m_{1}}-\frac{S_{\mathrm{f}}}{S_{\mathrm{f}, 0}}\left(\frac{\sigma}{\sigma_{02}}\right)^{m_{2}}\right\}
$$

where $L_{\mathrm{f}}$ is the gauge length, $S_{\mathrm{f}, 0}\left(=2 \pi R L_{\mathrm{f}, 0}\right)$ is the representative surface area of the fiber $\left(L_{\mathrm{f}, 0}=10 \mathrm{~mm}\right), \sigma_{01}$ and $\sigma_{02}$ are the Weibull scale parameters, and $m_{1}$ and $m_{2}$ are the Weibull shape parameters.

The fibers are hexagonally arranged in the full composite model based on the report by Tavares et al. (Tavares et al., 2017); thus, the fiber surface can be divided in the six segments. The surface stress concentration factor of the $i$-th fiber segment next to a broken fiber at the fracture plane was considered by assuming that it was $\alpha_{\mathrm{i}}$ times the fiber stress with no additional surface stress concentration $\sigma_{\mathrm{f}}$. The stress concentration factor $\alpha_{\mathrm{i}}$ is given as follows:

$$
\alpha_{\mathrm{i}}=1+\gamma_{\mathrm{i}}\left(1-\frac{D_{\mathrm{s}}}{l_{\mathrm{s}}}\right)
$$

where $\gamma_{i}$ is the additional stress concentration term, and $l_{\mathrm{s}}$ is the stress recovery length; thus considering of the stress concentration on an intact fiber surface, fiber breakage probability $P_{\mathrm{f}}(\sigma)$ can be rewritten as follows:

$$
P_{\mathrm{f}, \mathrm{i}}(\sigma)=1-\exp \left\{-\frac{S_{\mathrm{f}, \mathrm{i}}}{S_{\mathrm{f}, 0}}\left(\frac{\alpha_{\mathrm{i}} \sigma}{\sigma_{01}}\right)^{\mathrm{m}_{1}}-\frac{S_{\mathrm{f}, \mathrm{i}}}{S_{\mathrm{f}, 0}}\left(\frac{\alpha_{\mathrm{i}} \sigma}{\sigma_{02}}\right)^{\mathrm{m}_{2}}\right\}
$$

where $S_{\mathrm{f}, \mathrm{i}}$ is the $i$-th fiber segment of surface area $\left(S_{\mathrm{f}, \mathrm{i}}=\pi R^{2} L_{\mathrm{f}, 0} / 3\right)$. The strength of the $n$-th fiber segment is determined by choosing a random number $R_{\mathrm{n}}$ ranging from 0 to 1 and solving equation $R_{\mathrm{n}}=P_{\mathrm{f}, \mathrm{n}}\left(\sigma_{\mathrm{n}}\right)$. The longitudinal element was removed from the model when the stress applied to a fiber at the $n$-th fiber segment reached the statistical distribution of the fiber strength $\sigma_{\mathrm{n}}$.

The stress concentration factor on the intact fiber surface were determined by implementing the SEM, with the aim of ensuring that it was equivalent to the percentage of the coordinated fracture, which is defined as a failure occurring

Table 1 Bimodal Weibull parameter of the T1100G carbon fiber and two different types of PAN-based carbon fibers (i.e., T800S and T700S).

\begin{tabular}{l|c|c|c|c|c|c|c}
\hline \hline Types & $\sigma_{01}$ & $m_{1}$ & $\sigma_{02}$ & $m_{2}$ & $\sigma_{01} / \sigma_{02}$ & $m_{1} / m_{2}$ & Source \\
\hline T1100G & 7.7 & 4.5 & 9.1 & 13.0 & 0.85 & 0.35 & Yamamoto et al., 2019 \\
\hline $\mathrm{T} 800 \mathrm{~S}$ & 6.9 & 4.1 & 8.3 & 13.0 & 0.83 & 0.32 & Watanabe et al., 2018 \\
\hdashline $\mathrm{T} 700 \mathrm{~S}$ & 5.2 & 4.8 & 6.1 & 12.0 & 0.85 & 0.40 & Watanabe et al., 2018 \\
\hline
\end{tabular}


at the elements neighboring a broken fiber element in the horizontal plane of the broken fiber element, that was determined through the double-fiber fragmentation testing. Therefore, two longitudinal spring elements in the center of the SEM were assigned to the fibers, while the remaining longitudinal element were assigned to the matrix. The fiber elements with $10 \mathrm{~mm}$ in length were divided into 2000 segments (i.e., the unit length of the longitudinal spring elements was $5 \mu \mathrm{m}$ ). A coordination of fracture was defined as a failure that occurred at neighboring elements next to a broken fiber element in the same horizontal plane with respect to the broken fiber element.

\subsection{Experimental procedures}

High-strength, polyacrylonitrile (PAN)-based carbon fiber (TORAYCATM T1100G) and bisphenol-A epoxy resin materials were used to elaborate both the double-fiber fragmentation composites and the UD composites. The Young's modulus $\left(E_{\mathrm{f}}\right)$, tensile strength $\left(\sigma_{\mathrm{f}}\right)$, and diameter $(D)$ of the T1100G carbon fiber are $324 \mathrm{GPa}, 7.0 \mathrm{GPa}$, and $5.4 \mu \mathrm{m}$, respectively. Figure 1 and Table 2 present a summary of the mechanical properties of the epoxy materials. Note that all epoxy materials examined herein showed elastic-plastic transition behavior, exhibiting a stress-strain relationship that is typically observed in conventional epoxy materials.

Double-fiber fragmentation composites were elaborated by positioning two fibers parallel to the loading direction, implementing an interfiber spacing of approximately one-half to four fiber diameters. Figure 2(a) shows the schematic illustration of the fragmentation composite. The average interfiber spacing of seven fragmentation composites was approximately $10 \mu \mathrm{m}$. Next, a pre-heated and degassed epoxy resin was poured into a pre-heated glass model where the fibers were bonded. Subsequently, the fragmentation composites were cured in an air oven at temperature of $160^{\circ} \mathrm{C}$ for 5 hours prior to post-curing at temperature of $180^{\circ} \mathrm{C}$ for 2 hours. Finally, the composites were cooled in the oven to room temperature before being cut into rectangles with thickness of approximately $2.0 \mathrm{~mm}$ using a laboratory cut-off saw (metlon, METACUT 251).

The fragmentation composites were deformed in a four-point bending rig while the strain was monitored using strain gauge technique. The inner and outer span lengths for four-point bending tests were $18 \mathrm{~mm}$ and $50 \mathrm{~mm}$, respectively. The tensile strain was applied in tension until a maximum of $5.0 \%$ strain, and held constant at every $0.1 \%$ steps. As a fiber-fiber interaction criterion, fiber fractures that occurred at an angle within $0^{\circ}$ to $45^{\circ}$ are defined as coordinated fractures based on the nature of elastoplastic polymer material fracture phenomena (Huang et al., 2006). The number and position of broken fibers were recorded using the polarized-light microscope (OLYMPUS, BX60).

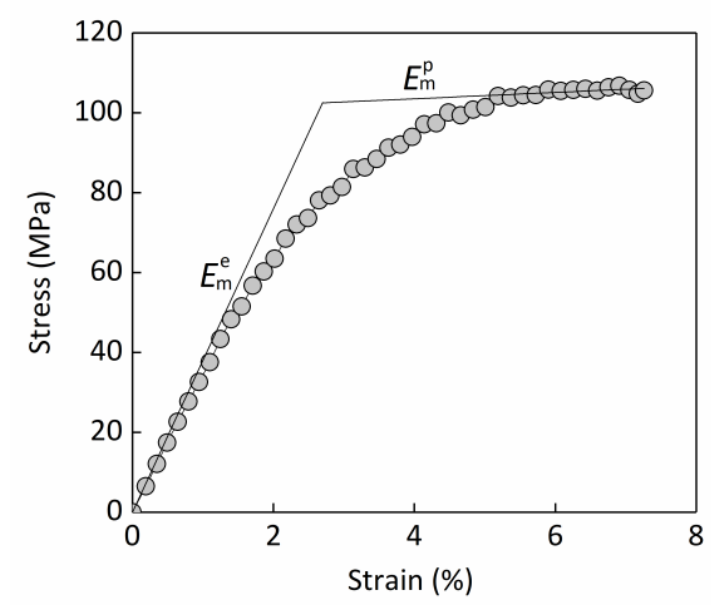

Fig. 1 Representative stress-strain curve for the epoxy materials.

Table 2 Material properties of the epoxy material.

\begin{tabular}{c|c|c|c}
\hline \hline $\begin{array}{l}\text { Young's modulus, } E_{\mathrm{m}}^{\mathrm{e}} \\
(\mathrm{GPa})\end{array}$ & $\begin{array}{l}\text { Tangent modulus, } E_{\mathrm{m}}^{\mathrm{p}} \\
(\mathrm{MPa})\end{array}$ & $\begin{array}{l}\text { Yield stress, } \sigma_{\mathrm{y}} \\
(\mathrm{MPa})\end{array}$ & $\begin{array}{l}\text { Shear modulus, } G_{\mathrm{m}} \\
(\mathrm{GPa})\end{array}$ \\
\hline 3.8 & 80 & 103 & 1.4 \\
\hline
\end{tabular}


(a)

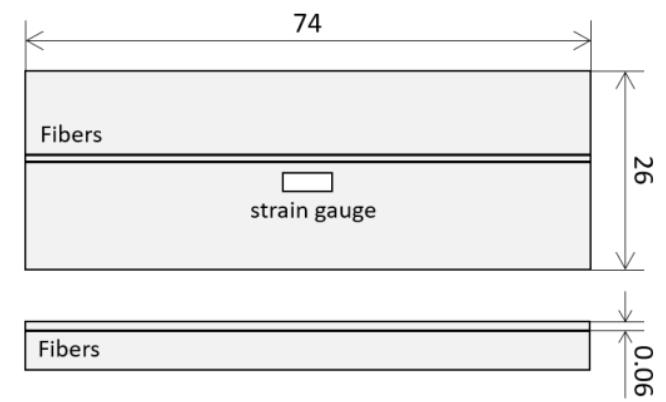

(b)

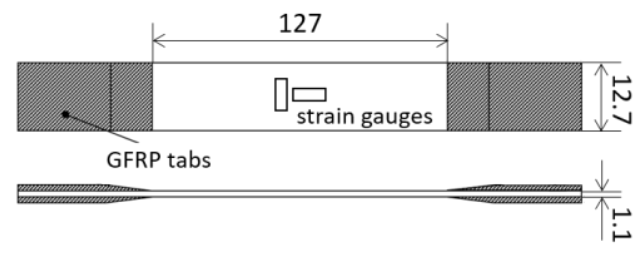

Fig. 2 Schematics of (a) the double-fiber fragmentation composite and (b) the UD composite.

The strain subjected to the carbon fiber $\varepsilon_{\mathrm{f}}$ was calculated as follows:

$$
\varepsilon_{\mathrm{f}}=\varepsilon_{\mathrm{c}} \times \frac{2.0}{\kappa} \times\left(\frac{t-2 d}{t}\right)-\varepsilon_{\mathrm{f}}^{\mathrm{r}},
$$

where $\varepsilon_{\mathrm{c}}$ is the strain of the recorded fragmentation composite, $\kappa$ is the strain gauge factor $(=2.13$; KYOWA, KFG-2-120-C1-11), $t$ and $d$ are the thickness of the fragmentation composite $(=\sim 2 \mathrm{~mm})$ and the embedded depth of the fibers $(=\sim 60 \mu \mathrm{m})$, respectively, $\varepsilon_{\mathrm{f}}^{\mathrm{r}}$ is strain originating from the difference coefficient of thermal expansion between fiber and matrix $(0.27 \%)$, as investigated through Raman spectrum analysis.

The tensile strength of the UD composite was investigated to validate the accuracy of the predicted tensile strength by the SEM derived based on the double-fiber fragmentation tests. The UD composites were elaborated through conventional bagging and autoclave laminating procedures to prepare the laminated structure with $\left[0_{6}\right]$. Figure $2(\mathrm{~b})$ shows the schematic illustration of the UD composite. The fiber volume fraction and the bulk density of the prepared UD composites were $57 \%$ and $1.79 \mathrm{Mg} / \mathrm{m}^{3}$, respectively. The tensile strengths were measured by the tensile-loading experiments under ambient conditions. A crosshead speed for the tensile experiments were $1.27 \mathrm{~mm} / \mathrm{min}(21.2 \mu \mathrm{m} / \mathrm{s})$. The strain gauges were used to measure the longitudinal and transverse strains of one side of the UD composites.

\section{Results and discussion 3.1 Tensile strength prediction}

First, we measured fiber fracture behavior in the double-fiber fragmentation composites to quantitatively determine the surface stress concentration factor. The investigations have demonstrated under the experimental conditions used in the study that matrix cracks and the coordination of fractures in adjacent fibers were observed, regardless of the interfiber spacing. Even though some variation has been observed for the percentages of coordinate fractures, the fragmentation tests performed herein revealed that different interfiber spacing does not apparent impact on the percentage of coordinated fractures. At $2.5 \%$ fiber strain $\varepsilon_{\mathrm{f}}$, calculated by the equation (11) for a composite strain $\varepsilon_{\mathrm{c}}$ of $5.0 \%$, the percentages of coordinated fracture was $9.09 \%$.

A quantitative determination of the stress concentration on the surface of an intact fiber around a fiber break point was conducted by implementing the SEM to investigate the value of stress concentration. Figure 3 depicts the relationship between the stress concentration factor and the percentage of coordinated fractures, which was determined by systematically sweeping stress concentration factor in the SEM simulation. The simulation results revealed that the percentage of coordinated fractures increased with the increasing stress concentration factors, and upon comparing the simulated percentages of coordinated fractures to the corresponding experimental results, the stress concentration factor on the surface of an intact fiber was estimated to be approximately 1.75 , indicating that 1.75 times as much concentrated stress subjected on the intact fiber surface as the fiber stress compared with no additional surface stress concentration conditions.

Figure 4 illustrates the fiber fracture behaviors on the final failure planes simulated through the SEM simulation. We defined the situation where the plane, including the most fiber breaks, as the final failure plane. A stress ratio of less than 1 shows that the fiber failure occurred near the final failure plane, on the other hand, a stress ratio of 0 depicts that 


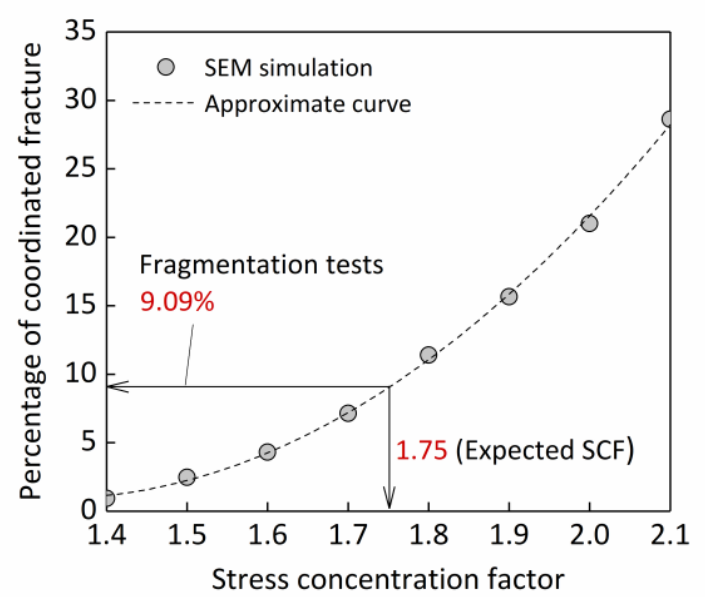

Fig. 3 Relationship between the stress concentration factors and the percentage of coordinated fractures.

the fiber failure occurred on the final failure plane. In the case of no additional stress concertation (i.e., $\alpha=1.0$ ), the fiber failure was clearly randomly distributed across the final failure plane, demonstrating that this is consistent with the statistical distribution of fiber strength. In contrast, additional stress concentration leads to the formation of broken fiber clusters, which results in relatively premature fracturing. Note that the experimentally obtained failure strain of the composites ranged from $1.93 \%$ to $2.01 \%$ (mean: $1.98 \%$ ), and the estimated failure strain simulated under the condition of $\alpha=1.75$ was approximately $2.20 \%(2.18 \%-2.21 \%)$; this value is roughly consistent with the experimentally obtained failure strain.

The tensile strength of the UD composite was predicted through the SEM technique. The model comprised 1024 fibers with $3 \mathrm{~mm}$ in length that were divided into 300 segments. The stress concentration was implemented to the intact fiber surface according to equation (9). Prior to comparing the simulated strengths to the experimental results, the simulated result were subjected to size scaling. This is given as follows (Okabe et al., 2002):

$$
n L=-n_{\mathrm{s}} L_{\mathrm{s}} / \ln \left\{1-F_{\mathrm{s}}\left(\tilde{\sigma}_{\mathrm{n}}\right)\right\}
$$

where $n_{\mathrm{s}}$ is the number of the fibers in the model $\left(n_{\mathrm{s}}=1024\right), L_{\mathrm{s}}$ is the length of the composite model $\left(L_{\mathrm{s}}=3 \mathrm{~mm}\right)$, and

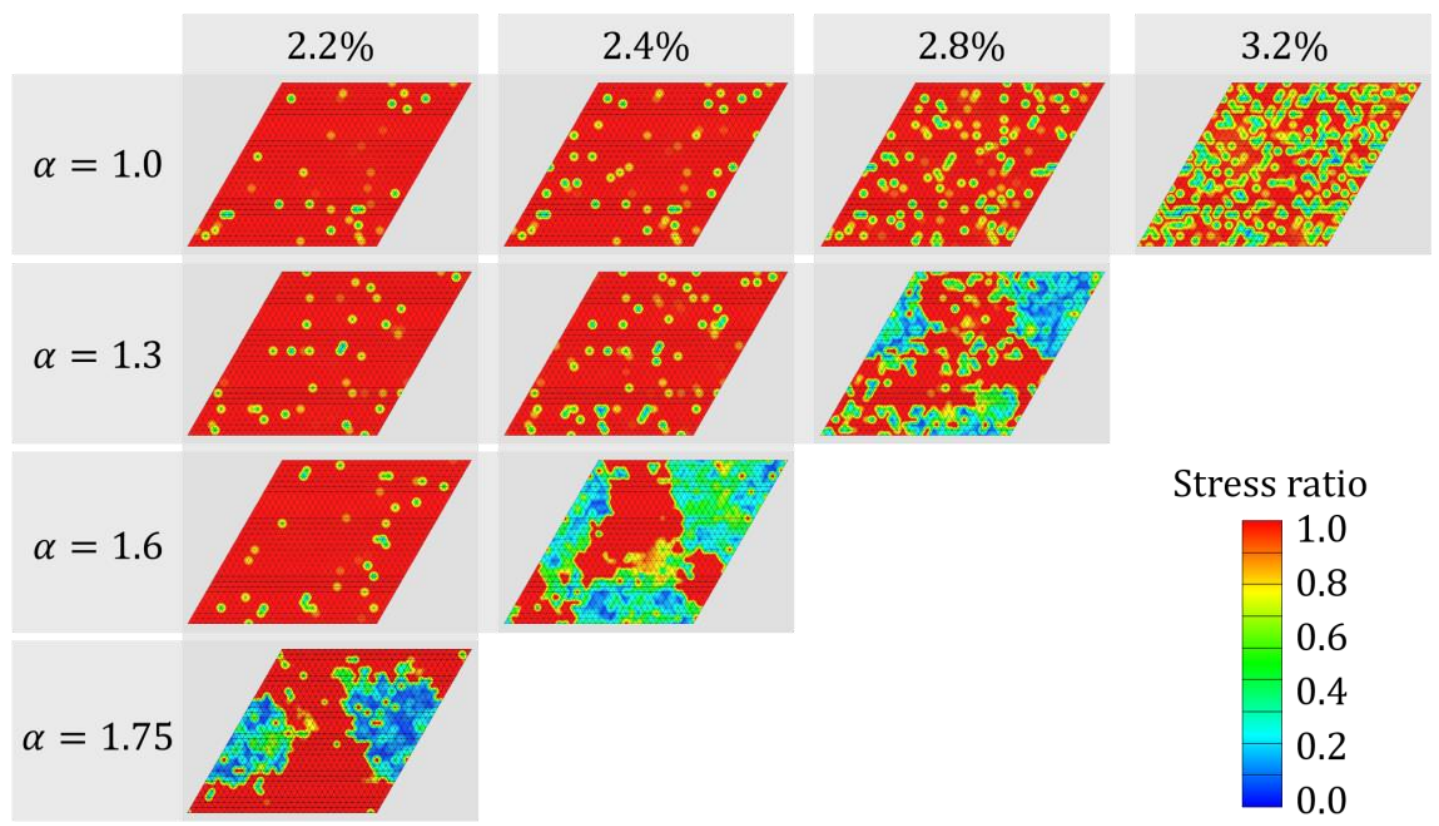

Fig. 4 Distribution of fiber stress in the axial direction on the final failure planes. 

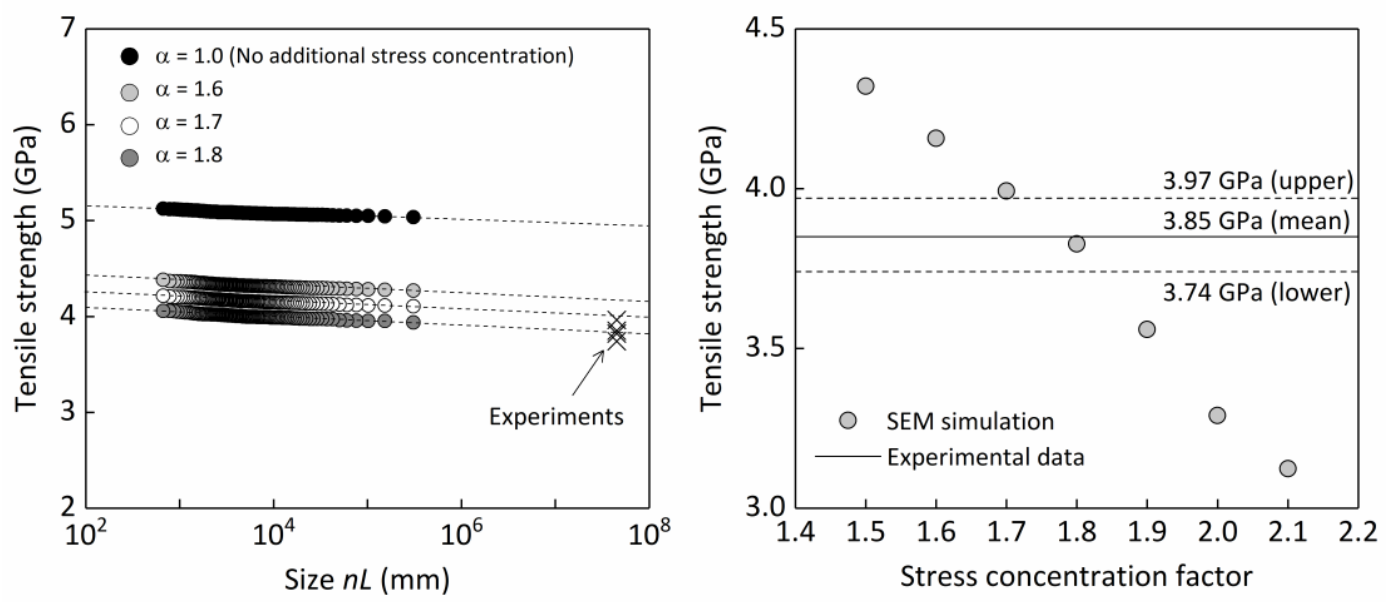

Fig. 5 Experimental and simulated results for the UD composites.

$F_{\mathrm{S}}\left(\tilde{\sigma}_{\mathrm{n}}\right)$ is the cumulative probability of failure at a given strength as determined 100 SEM simulation runs, and $n L$ is the size at which the characteristic strength is achieved. Figure 5 shows a comparison of the experimental and simulated results for the UD composites. In these figures, the circle symbols indicate the size scaled strength results calculated based to equation (12), while the crosses denote the experimentally obtained tensile strengths of these composites, which ranged from 3.74 to $3.97 \mathrm{GPa}$ (mean: $3.85 \mathrm{GPa}$ ). The simulated result obtained without consideration of additional stress concertation (i.e., $\alpha=1.0$ ) was incongruent with the experimental data, whereas the predictions incorporating the stress concentration factors of 1.7 and 1.8 were reasonably consistent with the experimental data. The estimated tensile strength obtained under the conditions of $\alpha=1.75$ was approximately $3.9 \mathrm{GPa}$. Furthermore, although the prediction with $\alpha=1.8$ seems to yield a better tensile strength prediction than with $\alpha=1.75$, we conclude that the prediction method proposed herein yields a reasonably accurate tensile strength prediction when the matrix crack-induced surface stress concentration of fibers is appropriately considered.

Improving the tensile strength prediction accuracy is central to CFRP composite research. As for one example, in certain types of CFRP material (Okabe et al., 2002), the model based on 3D shear-lag theory estimated overly the tensile strengths compared with the experimental data, and we could not make a full explanation of its reason. The results reported herein indicate that the fact that matrix cracks around the fiber break points increase the degree of stress concentrated on the intact fiber surface must be considered to obtain a high-accuracy strength prediction.

\subsection{Effects of bimodal Weibull scale parameters}

It can be seen from Table 1 that different types of the PAN-based carbon fibers possess different bimodal Weibull scale and shape parameters (Watanabe et al., 2018; Yamamoto et al., 2019). Interestingly, such fiber has unique $\sigma_{01}$ and $\sigma_{02}$ values, but their ratios (i.e., $\sigma_{01} / \sigma_{02}$ ) are approximately the same; that is $\sim 0.84$. On the basis of this fact that we applied the above-mentioned strength prediction procedures to investigate effects of the bimodal Weibull scale parameters on the tensile strength of the UD composites. Table 3 summarizes the parameters used to construct the

Table 3 Bimodal Weibull scale and shape parameters of the five different types of carbon fibers.

\begin{tabular}{|c|c|c|c|c|c|c|c|}
\hline Types & $\sigma_{01}$ & $m_{1}$ & $\sigma_{02}$ & $m_{2}$ & $\sigma_{01} / \sigma_{02}$ & $m_{1} / m_{2}$ & Remarks \\
\hline Fiber A & 9.2 & \multirow{5}{*}{4.5} & 10.9 & \multirow{5}{*}{13.0} & 0.84 & \multirow{5}{*}{0.35} & $20 \%$ improvement over reference data \\
\hline Fiber B & 8.6 & & 10.2 & & 0.84 & & $10 \%$ improvement over reference data \\
\hline T1100G & 7.7 & & 9.1 & & 0.85 & & Reference data \\
\hline T800S & 6.9 & & 8.3 & & 0.83 & & $10 \%$ reduction over reference data \\
\hline Fiber C & 6.2 & & 7.3 & & 0.85 & & $20 \%$ reduction over reference data \\
\hline
\end{tabular}



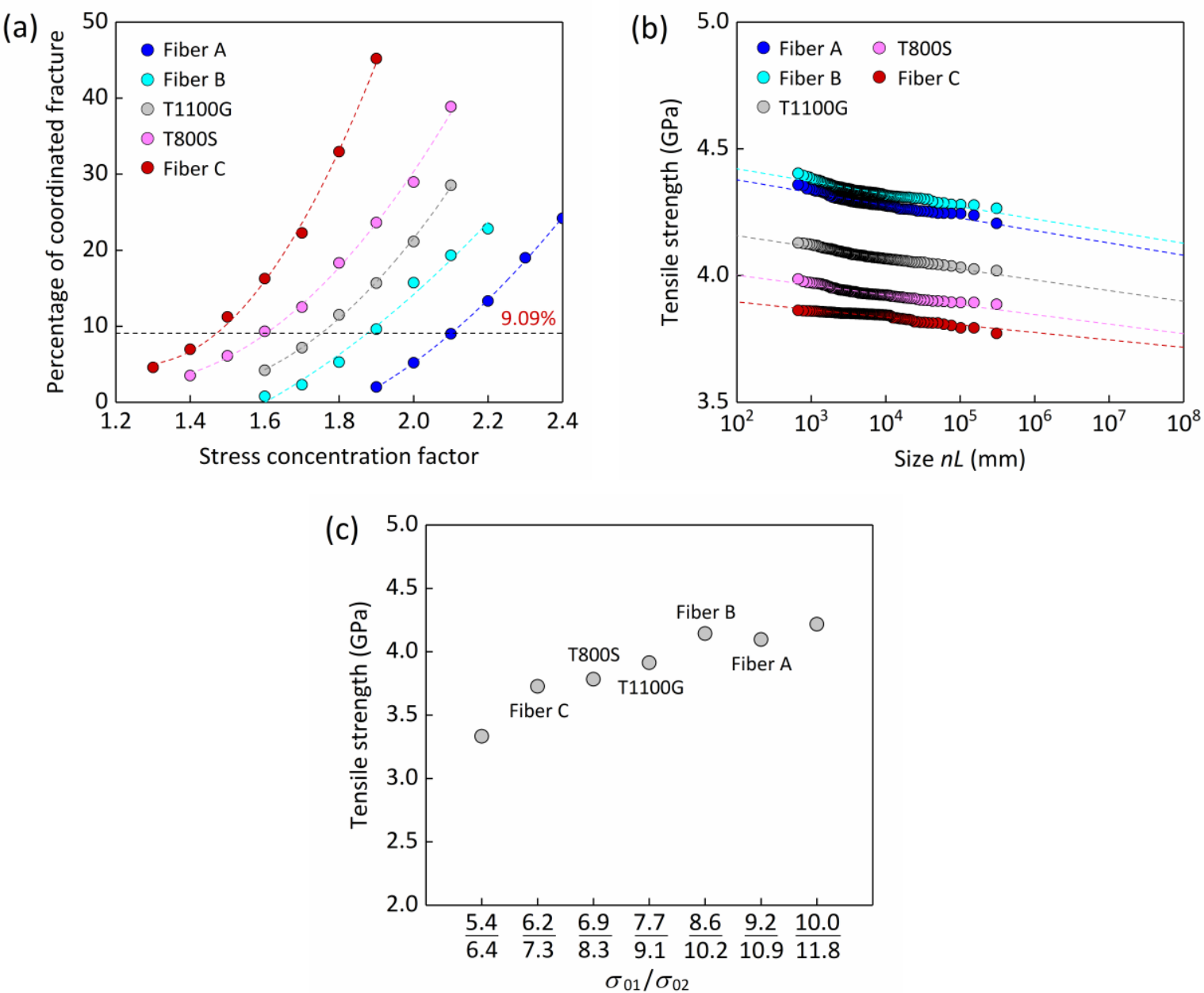

Fig. 6 (a) Relationship between the stress concentration factors and the percentage of coordinated fractures.

$(b, c)$ The scaled tensile strength results for the UD composites.

bimodal Weibull distribution for five different types of carbon fibers used in this section. We considered herein the situation where the all fiber failures have the Young's modulus of $324 \mathrm{GPa}$ and the diameter of $5.4 \mu \mathrm{m}$.

Figure 6(a) illustrates the results obtained through the SEM simulation to determine the stress concentration factors in each fibers. A comparison of the experimental and simulated results presented in Figure 6(a) revealed a clear difference in the estimated stress concentration factors. The percentages of coordinated fractures occurring in the fiber types of Fiber A, Fiber B, T1100G (reference data), T800S, and Fiber C were calculated to be 2.08, 1.88, 1.75, 1.61, and 1.46, respectively; an enhancement in the bimodal Weibull scale parameters leads to increase in the stress concentration factor. Thus, acquired surface stress concentration factors were implemented to predict the tensile strength of the UD composite. Figure 6(b) and 6(c) present the effects of the bimodal Weibull scale parameters on the tensile strength of the composites. The simulated results obtained by implementing the bimodal Weibull scale parameters $\left(\sigma_{01} / \sigma_{02}\right)$ of 5.4/6.4 and 10.0/11.8 are also indicated in the Figure 6(c). Contradictory to the results obtained in the section 3.1, (which revealed that higher stress concentration on an intact fiber surface results in relatively premature fracturing of UD composites), even if a larger stress concentration was occurring on intact fiber surfaces, increased tensile strength was observed for the UD composites with a larger stress concentration factor. Even though some variation was observed for the simulated results, the tensile strength of the series of the UD composites containing carbon fibers with $\sigma_{01} / \sigma_{02}$ of approximately 0.84 gently increased in response to the increase in the bimodal Weibull scale parameters.

Further evidence of the improved tensile strength was provided by the distribution of fiber stress in the axial direction on the final failure planes (Figure 7). It can be seen that a larger fracture strain is observed for the composite having a higher stress concentration factors, and the number of fiber breakage is small when comparing with the given 


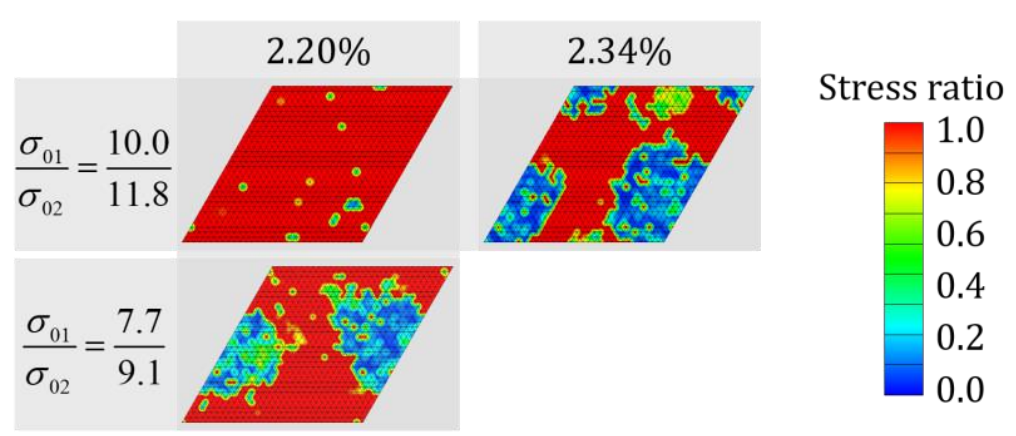

Fig. 7 Distribution of fiber stress in the axial direction on the final failure planes of the UD composite made with fibers having $\sigma_{01} / \sigma_{02}=10.0 / 11.8$ and $\sigma_{01} / \sigma_{02}=7.7 / 9.1$.

composite strain. These observations suggest that higher surface stress concentration does not necessarily mean that the relatively premature fracturing of the UD composite. But more than that enhanced mechanical integrity, namely the higher $\sigma_{01}$ and $\sigma_{02}$ values, of carbon fibers leads to the improved tensile strength of the UD composite, even though further detailed simulations are needed to be carried out in order to clarify the effects of the bimodal Weibull scale parameters on the tensile strength of the UD composite. The simulation results demonstrate that the present procedures, which implements the double-fiber fragmentation techniques in conjunction with SEM, may provide an effective route for development of UD composites with improved tensile strength, that it may be possible to tailor and control the bimodal Weibull scale parameters.

\subsection{Effects of bimodal Weibull shape parameters}

Next, we applied the strength prediction procedures to investigate the effects of the bimodal Weibull shape parameters on the tensile strength of the UD composites. Table 4 summarizes the parameters used to construct the bimodal Weibull distribution for five different types of carbon fiber used in this section. As in the previous section, we considered the situation where the all fiber failures possess the Young's modulus of $324 \mathrm{GPa}$ and the diameter of 5.4 $\mu \mathrm{m}$.

Figure 8(a) shows the results obtained through the SEM simulation to determine the stress concentration factors in each fibers; an increase in the bimodal Weibull shape parameters, i.e., $m_{1}$ and $m_{2}$, leads to increase in the stress concentration factor. The percentages of coordinated fractures occurring in the fiber types of Fiber D, Fiber E, T1100G (reference data), Fiber F, and Fiber $\mathrm{G}$ were estimated to be 1.59, 1.66, 1.75, 1.85, and 1.96, respectively. In the same manner with the section 3.2, the acquired surface stress concentration factors were implemented to predict the tensile strength of the UD composite.

Figure 8(b) and 8(c) show the effects of the bimodal Weibull shape parameters on the tensile strength of the UD composites; the tensile strength of the series of the UD composites containing carbon fibers with $m_{1} / m_{2}$ of approximately 0.35 increase approximately linearly in response to the increase in the bimodal Weibull shape parameters. This may be attributable to the fact that the larger values of the Weibull shape parameter indicate a smaller scatter of the fiber strength, therefore reduction in the strength distribution of the carbon fibers leads to the

Table 4 Bimodal Weibull scale and shape parameters of the five different types of carbon fibers.

\begin{tabular}{|c|c|c|c|c|c|c|c|}
\hline Types & $\sigma_{01}$ & $m_{1}$ & $\sigma_{02}$ & $m_{2}$ & $\sigma_{01} / \sigma_{02}$ & $m_{1} / m_{2}$ & Remarks \\
\hline Fiber D & \multirow{5}{*}{7.7} & 5.4 & \multirow{5}{*}{9.1} & 15.6 & \multirow{5}{*}{0.85} & 0.35 & $20 \%$ improvement over reference data \\
\hline Fiber E & & 5.0 & & 14.3 & & 0.35 & $10 \%$ improvement over reference data \\
\hline T1100G & & 4.5 & & 13.0 & & 0.35 & Reference data \\
\hline Fiber F & & 4.1 & & 11.7 & & 0.35 & $10 \%$ reduction over reference data \\
\hline Fiber $\mathrm{G}$ & & 3.6 & & 10.4 & & 0.35 & $20 \%$ reduction over reference data \\
\hline
\end{tabular}



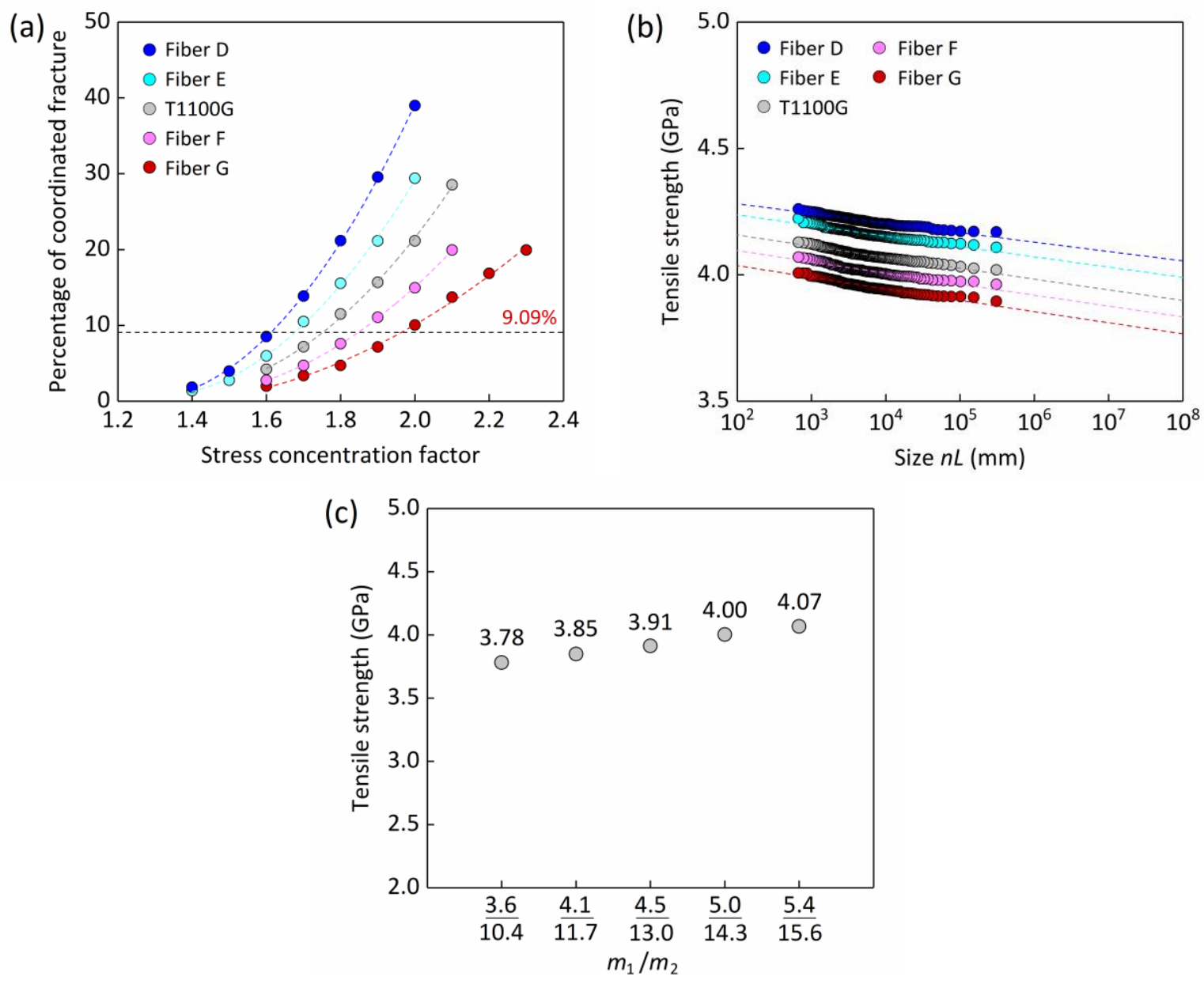

Fig. 8 (a) Relationship between the stress concentration factors and the percentage of coordinated fractures.

$(b, c)$ The scaled tensile strength results for the UD composites.

enhancement in the tensile strength of the UD composite. The carbon fiber with $m_{1} / m_{2}=5.4 / 15.6$ displays enhancement to the composite strength by factor of $\sim 1.08$ compared to the carbon fiber having $m_{1} / m_{2}=3.6 / 10.4$ (i.e., $50 \%$ shape parameter improved), indicating that the degree of enhancement in the tensile strength of the UD composite is limited.

\section{Conclusions}

The improvement of accuracy of prediction method for tensile strength has been a central focus of CFRP composite research. The results reported here indicated that the important factor for strength prediction with high accuracy will be considered an additional stress concentration caused by a fracture site in an adjacent fiber. We confirmed that employing the measured stress concentration factors and bimodal Weibull distribution to determine how strength is statistically distributed throughout the fiber yields the predicted strengths of the UD composite that are reasonably consistent with the experimental data, thereby demonstrating the validity of the proposed prediction method. We also demonstrated that the degree of stress concentrated on the surface of fibers can be changed by modifying the bimodal Weibull shape and scale parameters of carbon fibers. In totality, our findings provide a possible framework for guiding the creation of stronger CFRP composites. The design of such composites will need to account, or in some way circumvent, the stress concentration on the surface of fibers reported here.

\section{Acknowledgements}

This work was partly supported by Toray Industries, Inc., the Council for Science, Technology and Innovation(CSTI), the Cross-ministerial Strategic Innovation Promotion Program (SIP), and JSPS KAKENHI grant 
number 18K04721. The authors would like to acknowledge the vitally important encouragement and support made through the University of Washington-Tohoku University: Academic Open Space (UW-TU: AOS).

\section{References}

Huang, H. and Talreja, R., Numerical simulation of matrix micro-cracking in short fiber reinforced polymer composites: Initiation and propagation, Composites Science and Technology, Vol.66, No.15 (2006), pp.2743-2757.

Okabe, T. and Takeda, N., Elastoplastic shear-lag analysis of single-fiber composites and strength prediction of unidirectional multi-fiber composites, Composites Part A: Applied Science and Manufacturing, Vol.33, No.10 (2002), pp.1327-1335.

Okabe, T. and Takeda, N., Size effect on tensile strength of unidirectional CFRP composites experiment and simulation, Composites Science and Technology, Vol.62, No.15 (2002), pp.2053-2064.

Okabe, T., Sekine, H., Ishii, K., Nishikawa, M. and Takeda, N., Numerical method for failure simulation of unidirectional fiber-reinforced composites with spring element model, Composites Science and Technology, Vol.65, No.6 (2005), pp.921-933.

Scott, A.E., Mavrogordato, M., Wright, P., Sinclair, I. and Spearing, S.M., In situ fibre fracture measurement in carbon-epoxy laminates using high resolution computed tomography, Composites Science and Technology, Vol.71, No.21 (2011), pp1471-1477.

Swolfs, Y., McMeeking, R.M., Verpoest, I. and Gorbatikh, L., Matrix cracks around fibre breaks and their effect on stress redistribution and failure development in unidirectional composites, Composites Science and Technology, Vol.108 (2015), pp.16-22.

Tavares, R.P, Otero, F., Turon, A., Camanho, P.P., Effective simulation of the mechanics of longitudinal tensile failure of unidirectional polymer composites, International Journal of Fracture, Vol. 208, No.1-2 (2017), pp.269-285.

Watanabe, J., Tanaka, F., Okuda, H. and Okabe, T., Tensile strength distribution of carbon fibers at short gauge lengths, Advanced Composite Materials, Vol.23, No.1 (2014), pp.535-550.

Watanabe, J., Tanaka, F., Higuchi, R., Matsutani, H., Okuda, H. and Okabe, T., A study of stress concentrations around fiber breaks in unidirectional CF/epoxy composites using double-fiber fragmentation tests, Advanced Composite Materials, Vol.27, No.6 (2018), pp.575-587.

Yamamoto, G., Onodera, M., Watanabe, J., Okuda, H., Tanaka, F. and Okabe, T., Considering the stress concentration of fiber surfaces in the prediction of the tensile strength of unidirectional carbon fiber-reinforced plastic composites, Composites Part A: Applied Science and Manufacturing, Vol. 121 (2019), pp. 499-509. 